\title{
On the $L^{1}$ Stability to a Generalized Degasperis-Procesi Equation
}

\author{
Haibo Yan, ${ }^{1}$ Ls Yong, ${ }^{1}$ and Hanlei $\mathrm{Hu}^{2}$ \\ ${ }^{1}$ Department of Mathematics, Southwestern University of Finance and Economics, Chengdu 610074, China \\ ${ }^{2}$ Department of Mathematics, Sichuan Normal University, Chengdu 610066, China
}

Correspondence should be addressed to Ls Yong; yonglswang@163.com

Received 28 August 2013; Accepted 11 December 2013; Published 6 February 2014

Academic Editor: Yonghong Wu

Copyright (C) 2014 Haibo Yan et al. This is an open access article distributed under the Creative Commons Attribution License, which permits unrestricted use, distribution, and reproduction in any medium, provided the original work is properly cited.

A nonlinear generalized Degasperis-Procesi equation is investigated. Assuming that the strong solution of the equation is bounded in the sense of $L^{\infty}(R)$-norm and the initial data belong to the space $L^{1}(R) \cap L^{2}(R)$, we prove that the solutions are stable in the space $L^{1}(R)$.

\section{Introduction}

Coclite and Karlsen [1] investigated the following generalized Degasperis-Procesi equation:

$$
\begin{aligned}
u_{t}-u_{t x x}+4 f^{\prime}(u) u_{x}= & f^{\prime \prime \prime}(u) u_{x}^{3} \\
& +3 f^{\prime \prime}(u) u_{x} u_{x x}+f^{\prime}(u) u_{x x x} .
\end{aligned}
$$

When $f(u) \in C^{3}$ and satisfies

$$
\left|f^{\prime}(u)\right| \leq c|u|, \quad|f(u)| \leq c|u|^{2},
$$

or

$$
\left|f^{\prime}(u)\right| \leq c, \quad|f(u)| \leq c|u|
$$

where $c$ is a positive constant, the existence and $L^{1}$ stability of entropy weak solutions belonging to the class $L^{1}(R) \bigcap B V(R)$ are established for (1) in paper [1].

The objective of this paper is to study the generalized Degasperis-Procesi equation

$$
\begin{aligned}
u_{t}-u_{t x x}+m g^{\prime}(u) u_{x}= & g^{\prime \prime \prime}(u) u_{x}^{3} \\
& +3 g^{\prime \prime}(u) u_{x} u_{x x}+g^{\prime}(u) u_{x x x},
\end{aligned}
$$

where $m$ is a positive constant, $g(u)$ is a polynomial of order $n(n \geq 2)$, and $g(0)=0$. When $m=4$ and $g(u)=u^{2} / 2$,
(4) reduces to the classical Degasperis-Procesi model [2-10]. Assuming that there exists a strong solution to (4), which is bounded in its existence time interval $[0, T)$, and the initial value of (4) lies in $L^{1}(R) \cap L^{2}(R)$, we will prove that the strong solutions of the equation are stable in the space $L^{1}(R)$ (see Theorem 8 in Section 3). From the authors' knowledge, this is a new result for (4).

This paper is organized as follows. Section 2 gives several lemmas. The main result and its proof are presented in Section 3.

\section{Several Lemmas}

We consider the Cauchy problem of (4) in the following form:

$$
\begin{aligned}
u_{t}-u_{t x x}+m g^{\prime}(u) u_{x}= & g^{\prime \prime \prime}(u) u_{x}^{3} \\
& +3 g^{\prime \prime}(u) u_{x} u_{x x}+g^{\prime}(u) u_{x x x}, \\
u(0, x) & =u_{0}(x) .
\end{aligned}
$$

Applying the operator $\left(1-\partial_{x}^{2}\right)^{-1}$ to the first equation of problem (5), we obtain

$$
\begin{gathered}
u_{t}+g^{\prime}(u) u_{x}+\partial_{x} P_{u}=0, \\
u(0, x)=u_{0}(x)
\end{gathered}
$$


where $P_{u}=((m-1) / 2) \int_{R} e^{-|x-y|} g(u(t, y)) d y$. Letting $\Psi_{u}(u)=$ $\partial_{x} P_{u}$, we get

$$
\begin{gathered}
u_{t}+g^{\prime}(u) u_{x}+\Psi_{u}(u)=0, \\
u(0, x)=u_{0}(x) .
\end{gathered}
$$

Lemma 1. The solution of problem (5) with $m>0$ satisfies

$$
\int_{R} y_{1} y d x=\int_{R} \frac{1+\xi^{2}}{m+\xi^{2}}|\widehat{u}(\xi)|^{2} d \xi=\int_{R} \frac{1+\xi^{2}}{m+\xi^{2}}\left|\widehat{u_{0}}(\xi)\right|^{2} d \xi
$$

where $y_{1}=u-\partial_{x x}^{2} u$ and $y=\left(m-\partial_{x x}^{2}\right)^{-1} u$. Moreover, there exist two constants $c_{1}>0$ and $c_{2}>0$ depending only on $m$ such that

$$
c_{1}\left\|u_{0}\right\|_{L^{2}(R)} \leq c_{1}\|u\|_{L^{2}(R)} \leq c_{2}\left\|u_{0}\right\|_{L^{2}(R)} .
$$

Proof. Letting $y_{1}=u-\partial_{x x}^{2} u$ and $y=\left(m-\partial_{x x}^{2}\right)^{-1} u$ and using (4), we obtain $u=m y-y_{x x}$ and

$$
\begin{aligned}
\frac{d}{d t} \int_{R} y_{1} y d x & =\int_{R} \frac{\partial y_{1}}{\partial t} y d x+\int_{R} y_{1} \frac{\partial y}{\partial t} d x=2 \int_{R} \frac{\partial y_{1}}{\partial t} y d x \\
& =2 \int_{R}\left[-m g^{\prime}(u) u_{x}+g^{\prime \prime \prime}(u) u_{x}^{3}\right. \\
& \left.\quad+3 g^{\prime \prime}(u) u_{x} u_{x x}+g^{\prime}(u) u_{x x x}\right] y d x \\
& =2 \int_{R}\left[-m \partial_{x}[g(u)]+[g(u)]_{x x x}\right] y d x \\
& =\int_{R}[m g(u)] y_{x}-g(u) y_{x x x} d x \\
& =\int_{R}[m g(u)] y_{x}-g(u)\left(m y_{x}-u_{x}\right) d x \\
& =\int_{R} g(u) u_{x} d x, \\
& =0 .
\end{aligned}
$$

Using the Parseval identity and (10), we obtain (8) and (9).

Remark 2. When $m \leq 0$, from (8), we cannot obtain inequality (9).

Lemma 3. If $u_{0} \in L^{2}(R)$ and $\|u\|_{L^{\infty}(R)}<M$, it holds that

$$
\left\|P_{u}\right\|_{L^{\infty}\left(R_{+} \times R\right)}, \quad\left\|\Psi_{u}(u)\right\|_{L^{\infty}\left(R_{+} \times R\right)}<c_{0} M^{n-2},
$$

where $c_{0}$ is a constant independent of $t$ and $n \geq 2$.

Proof. Using the assumption $u_{0} \in L^{2}(R)$ and Lemma 1, we have $u \in L^{2}(R)$. Using (7), we get

$$
\begin{gathered}
P_{u}(t, x)=\frac{m-1}{2} \int_{R} e^{-|x-y|} g(u) d y, \\
\Psi_{u}(u(t, x))=\frac{m-1}{2} \int_{R} e^{-|x-y|} \operatorname{sign}(y-x) g(u) d y .
\end{gathered}
$$

Since the function $g(u)$ is a polynomial of order $n$ and $\|u\|_{L^{\infty}(R)}<M$, combining Lemma 1 derives that (11) holds.

Lemma 4. Assume that $\|u\|_{L^{\infty}(R)}<M$ and $\|v\|_{L^{\infty}(R)}<M$ are two solutions of (4) with initial data $u_{0}, v_{0} \in L^{2}(R)$, respectively. Then, for any $\phi(t, x) \in C_{0}^{\infty}([0, \infty) \times R)$, it holds that

$$
\int_{-\infty}^{\infty}\left|\Psi_{u}(u)-\Psi_{v}(v)\right||\phi(t, x)| d x \leq c_{0} \int_{-\infty}^{\infty}|u-v| d x,
$$

where $c_{0}>0$ depends on $m, n, M, \phi,\left\|u_{0}\right\|_{L^{2}(R)}$, and $\left\|v_{0}\right\|_{L^{2}(R)}$.

Proof. We have

$$
\begin{aligned}
& \int_{-\infty}^{\infty}\left|\Psi_{u}(u)-\Psi_{v}(v)\right||\phi(t, x)| d x \\
& \leq(m-1) \int_{-\infty}^{\infty}\left|\partial_{x} \Lambda^{-2}(g(u)-g(v))\right||\phi(t, x)| d x \\
& =\frac{|m-1|}{2}\left|\int_{-\infty}^{\infty} \int_{-\infty}^{\infty} e^{-|x-y|}\right| \operatorname{sign}(x-y)|| g(u) \\
& \quad-g(v)|d y| \phi(t, x)|d x| \\
& \leq c_{0} \int_{-\infty}^{\infty}|u-v| M^{n-2} d y\left|\int_{-\infty}^{\infty}\right| \phi(t, x)|d x| \\
& \leq c_{0} \int_{-\infty}^{\infty}|u-v| d y,
\end{aligned}
$$

which completes the proof.

We define $\delta(\sigma)$ as a function which is infinitely differentiable on $(-\infty,+\infty)$ such that $\delta(\sigma) \geq 0, \delta(\sigma)=0$ for $|\sigma| \geq 1$, and $\int_{-\infty}^{\infty} \delta(\sigma) d \sigma=1$. For any number $\varepsilon>0$, we let $\delta_{\varepsilon}(\sigma)=\delta\left(\varepsilon^{-1} \sigma\right) / \varepsilon$. Then we know that $\delta_{\varepsilon}(\sigma)$ is a function in $C^{\infty}(-\infty, \infty)$ and

$$
\begin{gathered}
\delta_{\varepsilon}(\sigma) \geq 0, \quad \delta_{\varepsilon}(\sigma)=0 \quad \text { if }|\sigma| \geq \varepsilon, \\
\left|\delta_{\varepsilon}(\sigma)\right| \leq \frac{c}{\varepsilon}, \quad \int_{-\infty}^{\infty} \delta_{\varepsilon}(\sigma)=1 .
\end{gathered}
$$

Assume that the function $v(x)$ is locally integrable on $(-\infty, \infty)$. We define an approximation function of $v$ as

$$
v^{\varepsilon}(x)=\frac{1}{\varepsilon} \int_{-\infty}^{\infty} \delta\left(\frac{x-y}{\varepsilon}\right) v(y) d y, \quad \varepsilon>0 .
$$

We get $v^{\varepsilon}(x) \rightarrow v(x)$ as $\varepsilon \rightarrow 0$ almost everywhere.

We state the concept of a characteristic cone. For any $R_{0}>$ 0 , we define $N>\max _{t \in[0, T]}\|u\|_{L^{\infty}}<\infty$. Let $\mho$ represent the cone $\left\{(t, x):|x|<R_{0}-N t, 0 \leq t \leq T_{0}=\min \left(T, R_{0} N^{-1}\right)\right\}$. We let $S_{\tau}$ represent the cross section of the cone $\mho$ by the plane $t=\tau, \tau \in\left[0, T_{0}\right]$. Set $K_{r+2 \rho}=\{x:|x| \leq r+2 \rho\}$, where $r>0, \rho>0$, and $\pi_{T}=[0, T] \times R$ for an arbitrary $T>0$. The space of all infinitely differentiable functions $\phi(t, x)$ with compact support in $[0, T] \times R$ is denoted by $C_{0}^{\infty}\left(\pi_{T}\right)$. 
Lemma 5 (see [11]). Let the function $v(t, x)$ be bounded and measurable in cylinder $\Omega_{T}=[0, T] \times K_{r}$. If $\rho \in(0, \min [r, T])$ and $\varepsilon \in(0, \rho)$, then the function

$$
\begin{aligned}
V \varepsilon= & \frac{1}{\varepsilon^{2}} \\
& \times \iiint \int_{\substack{|(t-\tau) / 2| \leq \varepsilon, \rho \leq(t+\tau) / 2 \leq T-\rho,|(x-y) / 2| \leq \mathcal{\varepsilon},|(x+y) / 2| \leq r-\rho}}|v(t, x)-v(\tau, y)| d x d t d y d \tau
\end{aligned}
$$

satisfies $\lim _{\varepsilon \rightarrow 0} V_{\varepsilon}=0$.

Lemma 6 (see [11]). Let $|\partial F(u) / \partial u|$ be bounded. Then the function

$$
H(u, v)=\operatorname{sign}(u-v)(F(u)-F(v))
$$

satisfies the Lipschitz condition in $u$ and $v$, respectively.

Using the methods presented in [11], we have the following result.

Lemma 7. If $u$ is a strong solution of problem (6), $\phi(t, x) \in$ $C_{0}^{\infty}\left(\pi_{T}\right)$, and $\phi(0, x)=0$, it holds that

$$
\begin{gathered}
\iint_{\pi_{T}}\left\{|u-k| \phi_{t}+\operatorname{sign}(u-k)[g(u)-g(k)] \phi_{x}\right. \\
\left.-\operatorname{sign}(u-k) \Psi_{u}(t, x) \phi\right\} d x d t=0,
\end{gathered}
$$

where $k$ is an arbitrary constant.

Proof. Let $\Phi(u)$ be a twice differential function on the line $-\infty<u<\infty$. We multiply the first equation of problem (6) by the function $\Phi^{\prime}(u) \phi(t, x)$, where $\phi(t, x) \in C_{0}^{\infty}\left(\pi_{T}\right)$. Integrating over $\pi_{T}$ and transferring the derivatives with respect to $t$ and $x$ to the test function $\phi$, for any constant $k$, we obtain

$$
\begin{gathered}
\iint_{\pi_{T}}\left\{\Phi(u) \phi_{t}+\left[\int_{k}^{u} \Phi^{\prime}(z) g^{\prime}(z) d z\right] \phi_{x}\right. \\
\left.-\Phi^{\prime}(u) \Psi_{u}(t, x) \phi\right\} d x d t=0,
\end{gathered}
$$

in which we have used $\int_{-\infty}^{\infty}\left[\int_{k}^{u} \Phi^{\prime}(z) g^{\prime}(z) d z\right] \phi_{x} d x=$ $-\int_{-\infty}^{\infty}\left[\Phi^{\prime}(u) g^{\prime}(u) u_{x}\right] \phi(t, x) d x$.

Integration by parts yields that

$$
\begin{aligned}
\int_{-\infty}^{\infty} & {\left[\int_{k}^{u} \Phi^{\prime}(z) g^{\prime}(z) d z\right] \phi_{x} d x } \\
= & \int_{-\infty}^{\infty}\left[\Phi^{\prime}(u)[g(u)-g(k)]\right. \\
& \left.-\int_{k}^{u}[g(z)-g(k)] \Phi^{\prime \prime}(z) d z\right] \phi_{x} d x .
\end{aligned}
$$

Let $\Phi^{\varepsilon}(u)$ be an approximation of the function $|u-k|$ and set $\Phi(u)=\Phi^{\varepsilon}(u)$. Using the properties of the $\operatorname{sign}(u-k)$, from (20) and (21), and sending $\varepsilon \rightarrow 0$, we have

$$
\begin{gathered}
\iint_{\pi_{T}}\left\{|u-k| \phi_{t}+\operatorname{sign}(u-k)[g(u)-g(k)] \phi_{x}\right. \\
\left.-\operatorname{sign}(u-k) \Psi_{u}(t, x) \phi\right\} d x d t=0,
\end{gathered}
$$

which completes the proof.

\section{Main Result}

Generally speaking, we cannot get the boundedness of strong solutions for problem (6). This is why we assume that the strong solutions of problem (6) possess boundedness in order to establish the $L^{1}$ stability for the problem. Now we state our main result as follows.

Theorem 8. Assume that there exist strong solutions $u$ and $v$ for problem (5) or (6). Let $T$ be the maximum existence time for the solutions. If $\|u\|_{L^{\infty}(R)}<M,\|v\|_{L^{\infty}(R)}<M$, and the initial data $u_{0}, v_{0} \in L^{1}(R) \cap L^{2}(R)$, it holds that

$$
\begin{aligned}
\| u(t, \cdot) & -v(t, \cdot) \|_{L^{1}(R)} \\
& \leq c e^{c t} \int_{-\infty}^{\infty}\left|u_{0}(x)-v_{0}(x)\right| d x, \quad t \in[0, T],
\end{aligned}
$$

where $c$ depends on $\left\|u_{0}\right\|_{L^{2}(R)},\left\|v_{0}\right\|_{L^{2}(R)}, M, T$, and the coefficients of polynomial $g(u)$.

Proof. For $\phi(t, x) \in C_{0}^{\infty}\left(\pi_{T}\right)$, we assume that $\phi(t, x)=0$ outside the cylinder

$$
\uplus=\{(t, x)\}=[\rho, T-2 \rho] \times K_{r-2 \rho}, \quad 0<2 \rho \leq \min (T, r) .
$$

Let

$$
\begin{aligned}
\psi & =\phi\left(\frac{t+\tau}{2}, \frac{x+y}{2}\right) \delta_{\varepsilon}\left(\frac{t-\tau}{2}\right) \delta_{\varepsilon}\left(\frac{x-y}{2}\right) \\
& =\phi(\cdots) \lambda_{\varepsilon}(*),
\end{aligned}
$$

where $(\cdots)=((t+\tau) / 2,(x+y) / 2)$ and $(*)=((t-\tau) / 2,(x-$ $y) / 2)$. The function $\delta_{\varepsilon}(\sigma)$ is defined in (15). Note that

$$
\psi_{t}+\psi_{\tau}=\phi_{t}(\cdots) \lambda_{\varepsilon}(*), \quad \psi_{x}+\psi_{y}=\phi_{x}(\cdots) \lambda_{\varepsilon}(*) .
$$

Following Kruzkov's device of doubling the variables presented in [11], from Lemma 7, and choosing $k=v(\tau, y)$, we have

$$
\begin{aligned}
\iiint \int_{\pi_{T} \times \pi_{T}}\{ & |u(t, x)-v(\tau, y)| \psi_{t} \\
& +\operatorname{sign}(u(t, x)-v(\tau, y)) \\
& \times(g(u(t, x))-g(v(\tau, y))) \psi_{x} \\
& +\operatorname{sign}(u(t, x)-v(\tau, y)) \\
& \left.\times \Psi_{u}(t, x) \psi\right\} d x d t d y d \tau=0 .
\end{aligned}
$$


Similarly, it has

$$
\begin{aligned}
\iiint \int_{\pi_{T} \times \pi_{T}}\{ & |v(\tau, y)-u(t, x)| \psi_{\tau} \\
& +\operatorname{sign}(v(\tau, y)-u(t, x)) \\
& \times(g(u(t, x))-g(v(\tau, y))) \psi_{y} \\
& +\operatorname{sign}(v(\tau, y)-u(t, x)) \\
& \left.\times \Psi_{v}(\tau, y) \psi\right\} d x d t d y d \tau=0 .
\end{aligned}
$$

It follows from (27) and (28) that

$$
\begin{array}{r}
0 \leq \iiint_{\pi_{T} \times \pi_{T}}\left\{|u(t, x)-v(\tau, y)|\left(\psi_{t}+\psi_{\tau}\right)\right. \\
+\operatorname{sign}(u(t, x)-v(\tau, y)) \\
\times(g(u(t, x))-g(v(\tau, y))) \\
\left.\times\left(\psi_{x}+\psi_{y}\right)\right\} d x d t d y d \tau \\
+\mid \iiint_{\pi_{T} \times \pi_{T}} \operatorname{sign}(u(t, x)-v(t, x)) \\
\quad \times\left(\Psi_{u}(t, x)-\Psi_{v}(\tau, y)\right) \\
\quad \times \psi d x d t d y d \tau \mid \\
=B_{1}+B_{2}+\left|\iiint \int_{\pi_{T} \times \pi_{T}} B_{3} d x d t d y d \tau\right| .
\end{array}
$$

We will prove the following inequality:

$$
\begin{aligned}
0 \leq \iint_{\pi_{T}}\left\{|u(t, x)-v(t, x)| \phi_{t}\right. \\
\quad+\operatorname{sign}(u(t, x)-v(t, x)) \\
\left.\quad \times(g(u(t, x))-g(v(t, x))) \phi_{x}\right\} d x d t \\
+\mid \iint_{\pi_{T}} \operatorname{sign}(u(t, x)-v(t, x)) \\
\quad \times\left[\Psi_{u}(t, x)-\Psi_{v}(t, x)\right] \phi d x d t \mid .
\end{aligned}
$$

We observe that the first two terms of inequality (29) can be represented in the form

$$
J_{\varepsilon}=J(t, x, \tau, y, u(t, x), v(\tau, y)) \lambda_{\varepsilon}(*) .
$$

From Lemma 6, we know that $J_{\varepsilon}$ satisfies the Lipschitz condition in $u$ and $v$, respectively. By the choice of $\phi$, we have $J_{\varepsilon}=0$ outside the region

$$
\begin{aligned}
\{(t, x ; \tau, y)\}= & \left\{\rho \leq \frac{t+\tau}{2} \leq T-2 \rho, \frac{|t-\tau|}{2} \leq \varepsilon,\right. \\
& \left.\frac{|x+y|}{2} \leq r-2 \rho, \frac{|x-y|}{2} \leq \varepsilon\right\},
\end{aligned}
$$

$$
\begin{gathered}
\iiint \int_{\pi_{T} \times \pi_{T}} J_{\varepsilon} d x d t d y d \tau \\
=\iiint \int_{\pi_{T} \times \pi_{T}}[J(t, x, \tau, y, u(t, x), v(\tau, y)) \\
-J(t, x, t, x, u(t, x), v(t, x))] \\
\quad \times \lambda_{\varepsilon}(*) d x d t d y d \tau \\
+\iiint \int_{\pi_{T} \times \pi_{T}} \quad \begin{array}{c}
\quad(t, x, t, x, u(t, x), v(t, x)) \\
\quad \times \lambda_{\varepsilon}(*) d x d t d y d \tau
\end{array} \\
=A_{11}(\varepsilon)+A_{12} .
\end{gathered}
$$

Considering the estimate $|\lambda(*)| \leq c / \varepsilon^{2}$ and the expression of function $A_{11}(\varepsilon)$, we have

$$
\begin{aligned}
& \left|A_{11}(\varepsilon)\right| \\
& \leq c\left[\varepsilon+\frac{1}{\varepsilon^{2}}\right. \\
& \left.\times \iiint \int_{\substack{|(t-\tau) / 2| \leq \mathcal{E}, \rho \leq(t+\tau) / 2 \leq T-\rho,|(x-y) / 2| \leq \mathcal{\varepsilon},|(x+y) / 2| \leq r-\rho}}|v(t, x)-v(\tau, y)| d x d t d y d \tau\right],
\end{aligned}
$$

where the constant $c$ does not depend on $\varepsilon$. Using Lemma 5, we obtain $A_{11}(\varepsilon) \rightarrow 0$ as $\varepsilon \rightarrow 0$. The integral $A_{12}$ does not depend on $\varepsilon$. In fact, substituting $t=\alpha,(t-\tau) / 2=\beta, x=\eta$, and $(x-y) / 2=\xi$ and noting that

$$
\int_{-\varepsilon}^{\varepsilon} \int_{-\infty}^{\infty} \lambda_{\varepsilon}(\beta, \xi) d \xi d \beta=1
$$

we have

$$
\begin{aligned}
A_{12}=2^{2} \iint_{\pi_{T}} J_{\varepsilon}(\alpha, \eta, \alpha, \eta, u(\alpha, \eta), v(\alpha, \eta)) \\
\quad \times\left\{\int_{-\varepsilon}^{\varepsilon} \int_{-\infty}^{\infty} \lambda_{\varepsilon}(\beta, \xi) d \xi d \beta\right\} d \eta d \alpha \\
=4 \iint_{\pi_{T}} J(t, x, t, x, u(t, x), v(t, x)) d x d t .
\end{aligned}
$$

Hence

$$
\begin{aligned}
& \lim _{\varepsilon \rightarrow 0} \iiint \int_{\pi_{T} \times \pi_{T}} J_{\varepsilon} d x d t d y d \tau \\
& \quad=4 \iint_{\pi_{T}} J(t, x, t, x, u(t, x), v(t, x)) d x d t .
\end{aligned}
$$


Since

$$
\begin{aligned}
& B_{3}=\operatorname{sign}(u(t, x)-v(\tau, y)) \\
& \quad \times\left(\Psi_{u}(t, x)-\Psi_{v}(\tau, y)\right) \phi \lambda_{\varepsilon}(*) \\
& =\overline{B_{3}}(t, x, \tau, y) \lambda_{\varepsilon}(*), \\
& \iiint \int_{\pi_{T} \times \pi_{T}} B_{3} d x d t d y d \tau \\
& =\iiint \int_{\pi_{T} \times \pi_{T}}\left[\overline{B_{3}}(t, x, \tau, y)-\overline{B_{3}}(t, x, t, x)\right] \\
& \quad+\iiint \int_{\pi_{T} \times \pi_{T}} \overline{B_{3}}(t, x, t, x) \lambda_{\varepsilon}(*) d x d t d y d \tau \\
& =A_{21}(\varepsilon)+A_{22},
\end{aligned}
$$

we obtain

$$
\begin{aligned}
& \left|A_{21}(\varepsilon)\right| \\
& \leq c\left(\varepsilon+\frac{1}{\varepsilon^{2}}\right. \\
& \left.\times \iiint \int_{\substack{|(t-\tau) / 2| \leq \varepsilon, \rho \leq(t+\tau) / 2 \leq T-\rho,|(x-y) / 2| \leq \varepsilon,|(x+y) / 2| \leq r-\rho}}\left|\Psi_{v}(t, x)-\Psi_{v}(\tau, y)\right| d x d t d y d \tau\right) .
\end{aligned}
$$

Using Lemma 5, we have $A_{21}(\varepsilon) \rightarrow 0$ as $\varepsilon \rightarrow 0$. Using (35), we have

$$
\begin{aligned}
A_{22}= & 2^{2} \iint_{\pi_{T}} \overline{I_{3}}(\alpha, \eta, \alpha, \eta, u(\alpha, \eta), v(\alpha, \eta)) \\
& \times\left\{\int_{-h}^{h} \lambda_{\varepsilon}(\beta, \xi) d \xi d \beta\right\} d \eta d \alpha \\
= & 4 \iint_{\pi_{T}} \overline{I_{3}}(t, x, t, x, u(t, x), v(t, x)) d x d t \\
= & 4 \iint_{\pi_{T}} \operatorname{sign}(u(t, x)-v(t, x)) \\
& \times\left(\Psi_{u}(t, x)-\Psi_{v}(t, x)\right) \phi(t, x) d x d t .
\end{aligned}
$$

From (33),(37), (39), and (40), we prove that inequality (30) holds.

Set

$$
\omega(t)=\int_{-\infty}^{\infty}|u(t, x)-v(t, x)| d x
$$

We define

$$
\theta_{\varepsilon}=\int_{-\infty}^{\sigma} \delta_{\varepsilon}(\sigma) d \sigma, \quad\left(\theta_{\varepsilon}^{\prime}(\sigma)=\delta_{\varepsilon}(\sigma) \geq 0\right)
$$

and choose two numbers $\rho$ and $\tau \in\left(0, T_{0}\right), \rho<\tau$. In (30), we choose

$$
\begin{gathered}
\phi=\left[\theta_{\varepsilon}(t-\rho)-\theta_{\varepsilon}(t-\tau)\right] \chi(t, x), \\
h<\min \left(\rho, T_{0}-\tau\right),
\end{gathered}
$$

where

$$
\chi(t, x)=\chi_{h}(t, x)=1-\theta_{h}(|x|+N t-R+h), \quad h>0 .
$$

When $h$ is sufficiently small, we note that function $\chi(t, x)=$ 0 outside the cone $\mho$ and $\phi(t, x)=0$ outside the set $\uplus$. For $(t, x) \in \mho$, we have the relations

$$
0=\chi_{t}+N\left|\chi_{x}\right| \geq \chi_{t}+N \chi_{x}
$$

Applying (41)-(45) and (30), we have the inequality

$$
\begin{aligned}
& 0 \leq \iint_{\pi_{T_{0}}}\left\{\left[\delta_{\varepsilon}(t-\rho)-\delta_{\varepsilon}(t-\tau)\right] \chi_{h}\right. \\
& \quad \times|u(t, x)-v(t, x)|\} d x d t \\
& +\int_{0}^{T_{0}} \int_{-\infty}^{\infty}\left[\theta_{\varepsilon}(t-\rho)-\theta_{\varepsilon}(t-\tau)\right] \\
& \quad \times\left|\left[J_{u}(t, x)-J_{v}(t, x)\right] \chi_{h}(t, x)\right| d x d t
\end{aligned}
$$

Using Lemma 4 and letting $h \rightarrow 0$ and $R_{0} \rightarrow \infty$, we obtain

$$
\begin{aligned}
0 \leq \int_{0}^{T_{0}}\left\{\left[\delta_{\varepsilon}(t-\rho)-\delta_{\varepsilon}(t-\tau)\right]\right. \\
\left.\quad \times \int_{-\infty}^{\infty}|u(t, x)-v(t, x)| d x\right\} d t \\
+c \int_{0}^{T_{0}}\left[\theta_{\varepsilon}(t-\rho)-\theta_{\varepsilon}(t-\tau)\right] \\
\quad \times \int_{-\infty}^{\infty}|u(t, x)-v(t, x)| d x d t .
\end{aligned}
$$

By the properties of the function $\delta_{\varepsilon}(\sigma)$ for $\varepsilon \leq \min \left(\rho, T_{0}-\right.$ $\rho$ ), we have

$$
\begin{aligned}
& \left|\int_{0}^{T_{0}} \delta_{\varepsilon}(t-\rho) \omega(t) d t-\omega(\rho)\right| \\
& \quad=\left|\int_{0}^{T_{0}} \delta_{\varepsilon}(t-\rho)\right| \omega(t)-\omega(\rho)|d t| \\
& \quad \leq c \frac{1}{\varepsilon} \int_{\rho-\varepsilon}^{\rho+\varepsilon}|\omega(t)-\omega(\rho)| d t \longrightarrow 0, \quad \text { as } \varepsilon \longrightarrow 0,
\end{aligned}
$$

where $c$ is independent of $\varepsilon$. Letting

$$
F(\rho)=\int_{0}^{T_{0}} \theta_{\varepsilon}(t-\rho) \omega(t) d t=\int_{0}^{T_{0}} \int_{-\infty}^{t-\rho} \delta_{\varepsilon}(\sigma) d \sigma \omega(t) d t,
$$


we get

$$
F^{\prime}(\rho)=-\int_{0}^{T_{0}} \delta_{\varepsilon}(t-\rho) \omega(t) d t \longrightarrow-\omega(\rho), \quad \text { as } \varepsilon \longrightarrow 0,
$$

from which we obtain

$$
F(\rho) \longrightarrow F(0)-\int_{0}^{\rho} \omega(\sigma) d \sigma, \quad \text { as } \varepsilon \longrightarrow 0
$$

Similarly, we have

$$
F(\tau) \longrightarrow F(0)-\int_{0}^{\tau} \omega(\sigma) d \sigma, \quad \text { as } \varepsilon \longrightarrow 0 .
$$

It follows from (51) and (52) that

$$
F(\rho)-F(\tau) \longrightarrow \int_{\rho}^{\tau} \omega(\sigma) d \sigma, \quad \text { as } \varepsilon \longrightarrow 0
$$

Sending $\rho \rightarrow 0$ and $\tau \rightarrow t$ and using

$$
\begin{aligned}
& |u(\rho, x)-v(\rho, x)| \leq\left|u(\rho, x)-u_{0}(x)\right| \\
& \quad+\left|v(\rho, x)-v_{0}(x)\right|+\left|u_{0}(x)-v_{0}(x)\right|
\end{aligned}
$$

from (47), (48), and (53)-(54), we have

$$
\begin{gathered}
\int_{-\infty}^{\infty}|u(t, x)-v(t, x)| d x \leq \int_{-\infty}^{\infty}\left|u_{0}-v_{0}\right| d x \\
+c_{0} \int_{0}^{t} \int_{-\infty}^{\infty}|u(t, x)-v(t, x)| d x d t .
\end{gathered}
$$

Applying the Gronwall inequality yields the desired result.

\section{Conflict of Interests}

The authors declare that there is no conflict of interests regarding the publication of this paper.

\section{Acknowledgment}

This work is supported by the Fundamental Research Funds for the Central Universities (JBK120504).

\section{References}

[1] G. M. Coclite and K. H. Karlsen, "On the well-posedness of the Degasperis-Procesi equation," Journal of Functional Analysis, vol. 233, no. 1, pp. 60-91, 2006.

[2] A. Degasperis and M. Procesi, "Asymptotic integrability," in Symmetry and Perturbation Theory, A. Degasperis and G. Gaeta, Eds., vol. 1, no. 1, pp. 23-37, World Scientific, Singapore, 1999.

[3] D. Henry, "Infinite propagation speed for the DegasperisProcesi equation," Journal of Mathematical Analysis and Applications, vol. 311, no. 2, pp. 755-759, 2005.

[4] Z. Lin and Y. Liu, "Stability of peakons for the DegasperisProcesi equation," Communications on Pure and Applied Mathematics, vol. 62, no. 1, pp. 125-146, 2009.
[5] H. Lundmark and J. Szmigielski, "Multi-peakon solutions of the Degasperis-Procesi equation," Inverse Problems, vol. 19, no. 6, pp. 1241-1245, 2003.

[6] Y. Liu and Z. Yin, "Global existence and blow-up phenomena for the Degasperis-Procesi equation," Communications in Mathematical Physics, vol. 267, no. 3, pp. 801-820, 2006.

[7] S. Lai and Y. Wu, "A model containing both the Camassa-Holm and Degasperis-Procesi equations," Journal of Mathematical Analysis and Applications, vol. 374, no. 2, pp. 458-469, 2011.

[8] J. Lenells, "Traveling wave solutions of the Degasperis-Procesi equation," Journal of Mathematical Analysis and Applications, vol. 306, no. 1, pp. 72-82, 2005.

[9] O. G. Mustafa, "A note on the Degasperis-Procesi equation," Journal of Nonlinear Mathematical Physics, vol. 12, no. 1, pp. 1014, 2005.

[10] Z. Yin, "Global weak solutions for a new periodic integrable equation with peakon solutions," Journal of Functional Analysis, vol. 212, no. 1, pp. 182-194, 2004.

[11] S. N. Kruzkov, "First order quasi-linear equations in several independent variables," Mathematics of the USSR-Sbornik, vol. 10, no. 2, pp. 217-243, 1970. 


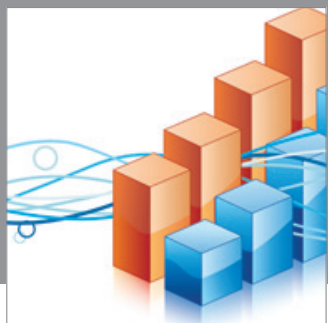

Advances in

Operations Research

mansans

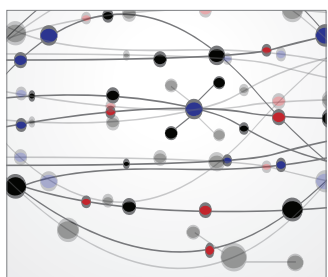

The Scientific World Journal
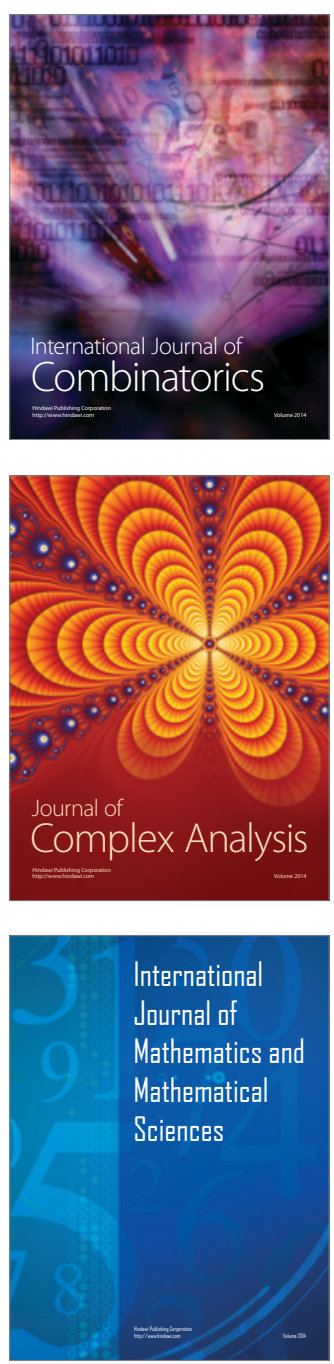
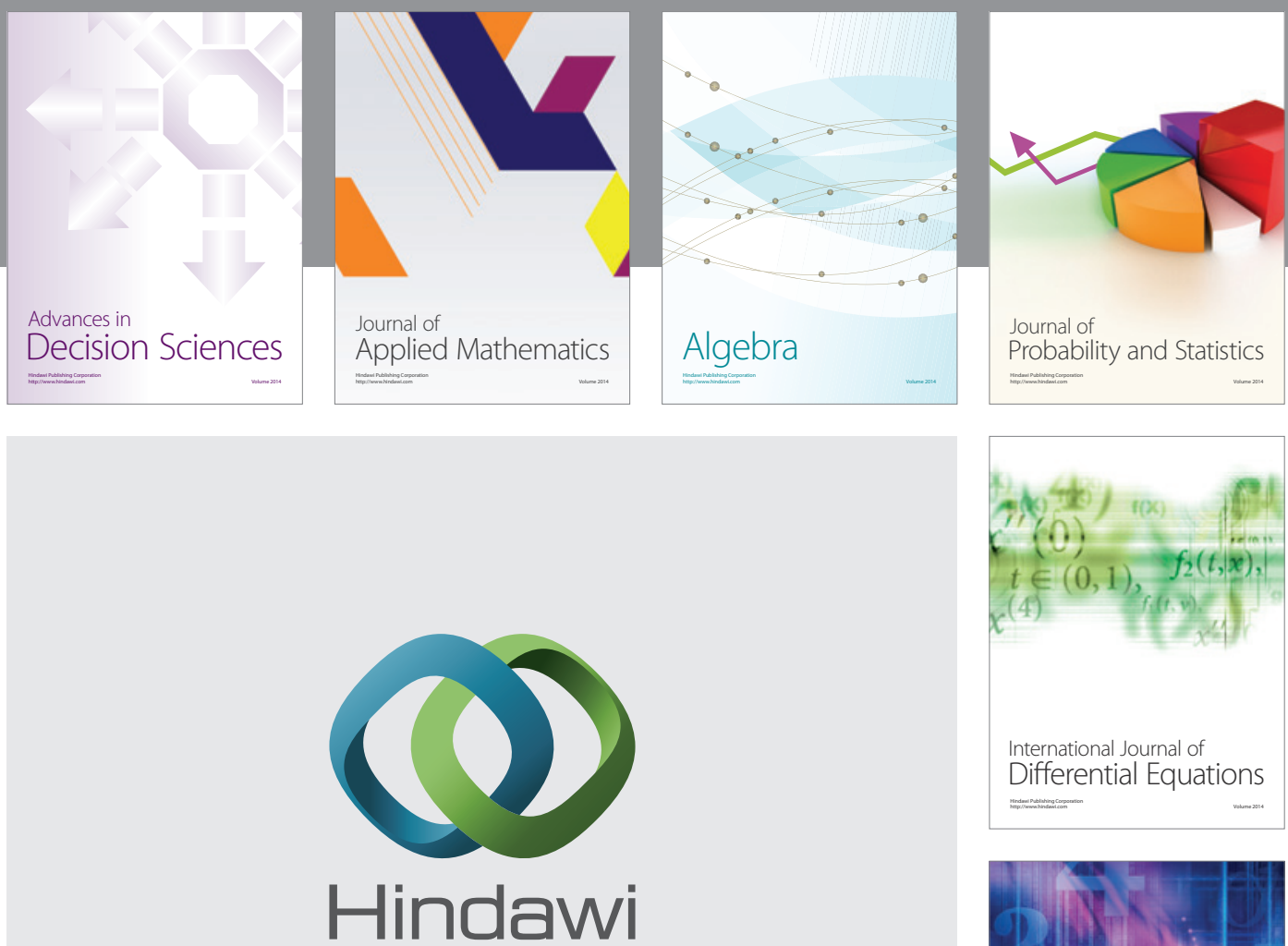

Submit your manuscripts at http://www.hindawi.com
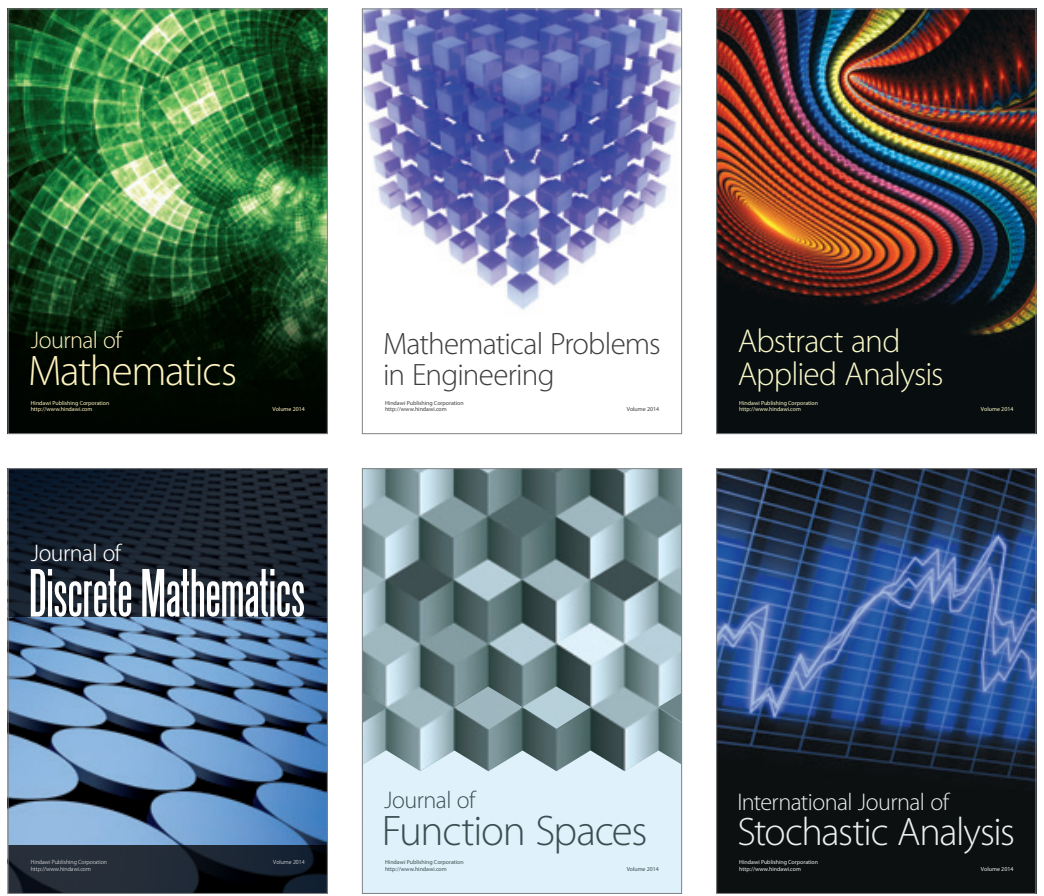

Journal of

Function Spaces

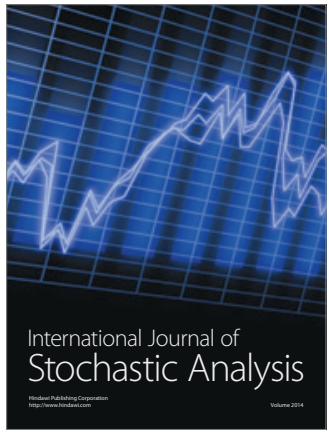

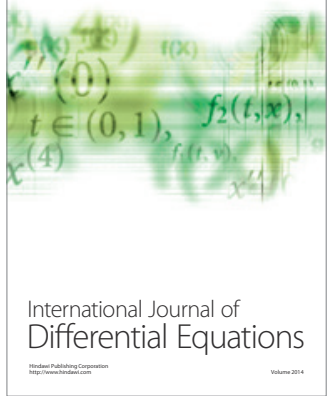
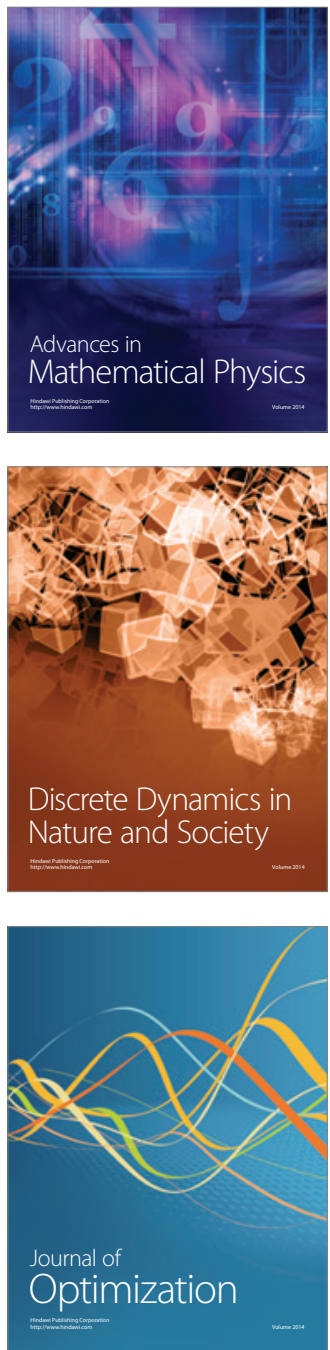